\title{
SELECTIVE REDUCTION OF ACTIVE METAL CHLORIDES FROM MOLTEN LICI-KCI USING LITHIUM DRAWDOWN
}

\author{
MICHAEL F. SIMPSON ${ }^{1 *}$, TAE-SIC YOO ${ }^{1}$, DANIEL LABRIER ${ }^{2}$, MICHAEL LINEBERRY ${ }^{2}$, \\ MICHAEL SHALTRY ${ }^{3}$, and SUPATHORN PHONGIKAROON ${ }^{3}$ \\ ${ }^{1}$ Idaho National Laboratory \\ Idaho National Laboratory, P.O. Box 1625, Idaho Falls, ID 83415 \\ ${ }^{2}$ Idaho State University \\ Campus Box 8060, Pocatello, ID 83209 \\ ${ }^{3}$ University of Idaho \\ 1776 Science Center Drive, Idaho Falls, Idaho 83402 \\ ${ }^{*}$ Corresponding author. E-mail : michael.simpson@inl.gov
}

Received February 09, 2011

Accepted for Publication November 14, 2011

In support of optimizing electrorefining technology for treating spent nuclear fuel, lithium drawdown has been investigated for separating actinides from molten salt electrolyte. Drawdown reaction selectivity is a major issue that requires investigation, since the goal is to remove actinides while leaving the fission products and other components in the salt. A series of lithium drawdown tests with surrogate fission product chlorides was run to obtain selectivity data with non-radioactive salts, develop a predictive model, and draw conclusions about the viability of using this process with actinide-loaded salt. Results of tests with $\mathrm{CsCl}, \mathrm{LaCl}_{3}, \mathrm{CeCl}_{3}$, and $\mathrm{NdCl}_{3}$ are reported here. Equilibrium was typically achieved in less than 10 hours of contact between lithium metal and molten salt under well-stirred conditions. Maintaining low oxygen and water impurity concentrations $(<10$ ppm) in the atmosphere was observed to be critical to minimize side reactions and maintain stable salt compositions. An equilibrium model has been formulated and fit to the experimental data. Good fits to the data were achieved. Based on analysis and results obtained to date, it is concluded that clean separation between minor actinides and lanthanides will be difficult to achieve using lithium drawdown.

KEYWORDS : Pyroprocessing, Drawdown, Lithium Reduction, Lanthanides, Electrorefining

\section{INTRODUCTION}

An advanced pyrochemical process for recycling nuclear fuel can be envisioned in which waste minimization, cost minimization, and high actinide recycle efficiency are all essential features. To achieve high actinide recycle efficiency, it is necessary to keep the actinides out of the waste streams. In the current implementation of pyroprocessing for treating spent fuel from Experimental Breeder Reactor-II (EBR-II) at Idaho National Laboratory (INL), waste salt from electrorefining is planned to be disposed of in a ceramic waste form consisting of glassbonded sodalite $[1,2]$. Since the salt contains up to 10 $\mathrm{wt} \%$ actinide chlorides, this represents a significant path for loss of the actinides into a waste stream. To avoid such loss, an additional step known as drawdown can be used to remove the actinides from the salt prior to treatment or disposal. The flow sheet shown in Fig. 1 depicts how drawdown can be implemented in a pyrochemical treatment process to achieve the simultaneous objectives of high actinide recycle and waste volume minimization.

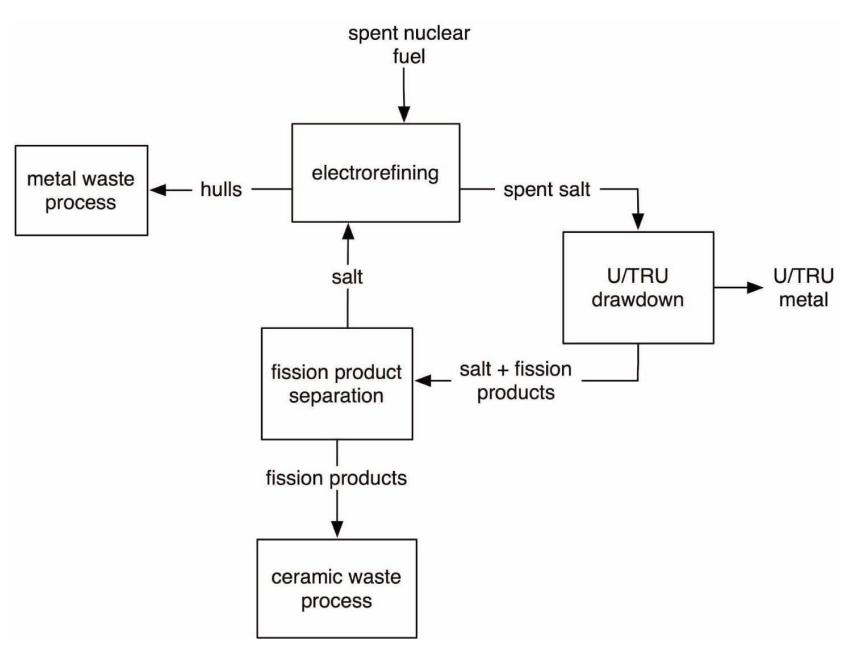

Fig. 1. Pyrochemical Process for Treating Spent Nuclear Fuel Featuring Drawdown for Improved Actinide Recycle and Waste Minimization. 
Salt that consists primarily of $\mathrm{LiCl}-\mathrm{KCl}$ must be periodically removed from the electrorefiner to reduce concentrations of these contaminants and facilitate continued electrochemical processing of spent fuel. Plutonium is one of the critical limiting contaminants that can dictate the need for treatment or disposal of the salt. The ability to selectively remove the actinides from the salt enables reduction of actinide concentration in the electrorefiner while keeping those actinides out of the waste stream. Post-drawdown, various separations methods can be used to remove rare earths and other active metals from the salt. The purified salt, consisting primarily of $\mathrm{LiCl}-\mathrm{KCl}$, can then be returned to the electrorefiner.

Reactive extraction into molten metals has been previously studied for achieving drawdown [3-8]. For example, researchers at Central Research Institute for Electric Power Industry (CRIEPI) in Japan investigated reactive extraction from $\mathrm{LiCl}-\mathrm{KCl}$, using lithium dissolved in liquid cadmium and bismuth [3,4]. Kurata et. al. first reported distribution coefficients for $\mathrm{U}, \mathrm{Np}$, and several rare earth (RE) elements between $\mathrm{LiCl}-\mathrm{KCl}$ and both liquid cadmium and liquid bismuth [4]. The results for cadmium were similar to those reported by Ackerman and Settle who had studied reactive extraction of transuranics via reaction with $U$ dissolved in cadmium [5], while bismuth exhibited an order of magnitude higher separation factors relative to $\mathrm{U}$. This likely led the CRIEPI researchers to further investigate the $\mathrm{LiCl}-\mathrm{KCl} / \mathrm{Bi}$ extraction system. Research reported by Kinoshita et al. focused on multi-stage extraction of transuranics into the liquid $\mathrm{Bi}$ phase [3]. Each extraction was run for about 4-6 hours. After a 5-stage extraction process, recovery of actinides was reported as greater than $99 \%$ for $\mathrm{U}, \mathrm{Pu}, \mathrm{Np}$, and $\mathrm{Am}$. Undesired extraction of REs (Ce, $\mathrm{Pr}, \mathrm{Nd}, \mathrm{La})$ also occurred at levels of approximately $7-20 \%$. The experimental results reported by Kinoshita et. al. agreed well with calculations based on the single-stage extraction experiments. Simulation of a $\mathrm{LiCl}-\mathrm{KCl} / \mathrm{Bi}$ counter-current extraction system predicted that a favorable ratio of TRU to RE could be accomplished, which is beneficial for both fuel fabrication and waste management [3].

Following up on previous experimental work by CRIEPI, Kobayashi of Japan Atomic Energy Agency (JAEA) performed an assessment of multi-stage countercurrent reactive extraction of the $\mathrm{Cd} / \mathrm{LiCl}-\mathrm{KCl}$ system [6]. It was found that at least 4 stages were necessary to recover $99 \%$ of the TRU from the salt with a decontamination factor higher than 5. It was also shown to be very difficult to separate $\mathrm{Np}, \mathrm{Am}$, or $\mathrm{Cm}$ from $\mathrm{Pu}$ due to the similarity in their separation factors. A similar analysis was recently published by Yoo et. al. from Korea Atomic Energy Research Institute (KAERI) in Republic of Korea [7]. For that paper, the CRIEPI data for both cadmium and bismuthbased systems were used as the basis for calculations. The stated objective was to develop a tool to assist in the design of actinide separations processes. As was the case with Kinoshita et. al., KAERI focused on a counter-current reductive extraction system. Via simulation, they investigated the effect of the amount of reducing agent in the molten metal, the number of stages in the process, and the salt/metal ratio. The Li content in the molten metal was found to be the most significant factor that affected the distribution of rare earths and actinides between the salt and metal phases.

Engineering concepts for reactive extraction have also been investigated. The high-temperature centrifugal contactor (i.e., pyrocontactor) developed by Argonne National Laboratory is an example of such a concept. Chow et al. described the operation of a pyrocontactor that extracts REs from electrorefiner salt while the salt is still in use by the electrorefiner, thereby eliminating the need to pause $U$ recovery operations to remove rare earths from the molten salt [8]. Development of pyrocontactors ended near the time of the shift from the Integral Fast Reactor (IFR) program to the EBR-II Spent Fuel Treatment program (c. 1994). This coincided with an overall deemphasis on development of drawdown technology, as it was seen as acceptable to let the actinides partition into the EBR-II ceramic waste forms.

The problem with reactive extraction is that it requires complicated and costly processing equipment. Molten metals and salts must be pumped through multiple stages of the extraction system at elevated temperatures (about $500^{\circ} \mathrm{C}$ ) using remotely-operated equipment in an inert atmosphere hot cell. This equipment is anticipated to be expensive to build, operate, and maintain. It would be easier to directly add lithium or other reactive metals to a bath of molten salt, allow the actinides to precipitate out of the salt as metals, send actinide-free salt to the waste process, and collect the U/TRU metal precipitate for new fuel fabrication. The chemistry of Li drawdown can be represented by the following reaction:

$$
3 \mathrm{Li}+\mathrm{MCl}_{3} \rightarrow \mathrm{M}+3 \mathrm{LiCl}
$$

where $\mathrm{M}$ represents an arbitrary actinide element $(\mathrm{U}, \mathrm{Pu}$, $\mathrm{Np}, \mathrm{Am}, \mathrm{Cm}$, etc.). This reaction is thermodynamically spontaneous, since the free energy change of reaction is negative for several active metals including $\mathrm{Li}, \mathrm{K}, \mathrm{Na}$, and $\mathrm{Mg}$. Based on Gibbs free energy of formation data, it is predicted that $U$ will be the first to be reduced in the salt followed by transuranic (TRU) elements followed by rare earth elements followed by Group I and II elements. Thus, in theory, it should be possible to remove the actinides while leaving the other fission products in the salt for subsequent separation and partitioning into the waste stream. Once reduced to metals, the actinides should precipitate with little to no solubility in the molten $\mathrm{LiCl}-\mathrm{KCl}$ at $500^{\circ} \mathrm{C}$.

Despite the apparent simplicity of using lithium reduction for drawdown, there are several unknowns related to this process-including reaction kinetics, equilibrium extent of reaction, and drawdown selectivity 
for the species of interest. As a first step in studying this system and the proposed method of direct lithium addition to electrorefiner salt, a series of experiments was performed with only molten $\mathrm{LiCl}-\mathrm{KCl}$ and non-actinide chlorides $\left(\mathrm{CsCl}, \mathrm{LaCl}_{3}, \mathrm{CeCl}_{3}\right.$, and $\left.\mathrm{NdCl}_{3}\right)$. The ability to selectively drawdown specific rare earths from the salt using direct lithium addition was tested to provide preliminary insight into the feasibility of this process for effectively performing actinide drawdown. Non-actinides were selected for the initial phase of the study, since the available experimental facilities were not yet approved for operations involving actinides. It was assessed that fundamental insight could be developed using rare earths and considering one of them a surrogate for an actinide. Transient data were collected, equilibrium states were approximated, and selectivity was measured and modeled.

\section{EXPERIMENTAL METHOD}

Anhydrous molten salt mixtures were prepared, heated, reacted, and sampled in an argon atmosphere glovebox. Two different glove boxes were used-one that utilized an argon purge with measured $\mathrm{O}_{2}$ concentrations of 20-40 $\mathrm{ppm}$, and one that used argon and a purification system to keep the impurities at less than $10 \mathrm{ppm}$ (both $\mathrm{O}_{2}$ and water). The salt mixtures consisted of 95 to $99 \mathrm{~mol} \%$ $\mathrm{LiCl}-\mathrm{KCl}$ in a eutectic composition $(58 \mathrm{~mol} \% \mathrm{LiCl})$ combined with two other active metal salts $\left(\mathrm{CsCl}-\mathrm{LaCl}_{3}\right.$, $\mathrm{CeCl}_{3}-\mathrm{LaCl}_{3}$ or $\mathrm{NdCl}_{3}-\mathrm{LaCl}_{3}$ ). The active metal salts were present in roughly the same concentrations based on mole percent. All salts were obtained from Sigma-Aldrich and had a purity of $>99.99 \%$. The salt was melted and homogenized by heating it to $500^{\circ} \mathrm{C}$ and sustaining this temperature overnight. Salts were contained in stainless steel or magnesia crucibles (5.1 to $8.9 \mathrm{~cm}$ diameter). Once in the molten state, the salt was continuously mixed using a submerged stirrer blade at $120 \mathrm{rpm}$.

Prior to lithium addition, an initial salt sample was taken using a threaded steel rod that was dipped into the salt to verify salt composition. A stainless steel tube $(1.27 \mathrm{~cm}$ diameter) inserted into the salt was then used for adding lithium. Initially, this tube rested on the bottom of the crucible. Pre-weighed lithium metal beads (99\% purity) were dropped into the tube. The tube was then pulled up slightly off the bottom of the crucible. This left the bottom of the tube in between the level of the top surface of the salt and the bottom of the crucible. Molten lithium could start diffusing into and reacting with the molten salt mixture. Salt samples $(0.15-0.25 \mathrm{~g})$ were taken nominally every hour for the first few hours after addition of the lithium. Stirring was not stopped to take samples. These samples were analyzed for elemental composition using inductively coupled plasma mass spectrometry (ICP-MS) with estimated error of $10 \%$. Equilibrium reaction conditions were estimated based on transient concentration measurements.

\section{MODEL DEVELOPMENT}

Consider a simple molten salt system consisting of two metal chlorides dissolved in eutectic $\mathrm{LiCl}-\mathrm{KCl}$. Addition of Li metal will result in reduction of one or both of these chlorides to the zero oxidation state- -assuming these salts are less thermodynamically stable than $\mathrm{LiCl}$. To model this reaction, two stages were constructed. In the first stage, the lithium is assumed to completely react with the least stable salt (based on free energy of formation of chloride salts from literature). If more lithium is available to react, the remaining amount is assumed to completely react with the second least stable salt. Consider the example of $\mathrm{LaCl}_{3}$ and $\mathrm{CeCl}_{3}$ being simultaneously reacted with lithium. Lanthanum chloride has a free energy of formation of $293.8 \mathrm{~kJ} / \mathrm{mole} \mathrm{Cl}$ at $500^{\circ} \mathrm{C}$ [9]. Cerium chloride is slightly less stable with a free energy of formation of $-288.0 \mathrm{~kJ} /$ mole $\mathrm{Cl}$ at the same temperature [9]. In this model, all of the lithium would initially react with $\mathrm{CeCl}_{3}$ to form $\mathrm{Ce}$ and $\mathrm{LiCl}$ until all of the $\mathrm{CeCl}_{3}$ was consumed. Any remaining $\mathrm{Li}$ would react with $\mathrm{LaCl}_{3}$. Using these assumptions, an initial non-equilibrium state can be calculated that includes elemental metals and their chloride salts. It is assumed that the lithium completely reacts and is no longer in the chemical system.

In the second stage of the model, the equilibrium point of the reaction between chloride salts and metals must be determined. From the above example, the equilibrium reaction to be calculated is as follows.

$$
\mathrm{LaCl}_{3}+\mathrm{Ce} \leftrightarrow \mathrm{CeCl}_{3}+\mathrm{La}
$$

It is assumed that a perfect separation between the two metal chlorides is impossible, so the above reaction will proceed to some extent in order to achieve equilibrium. This is a convenient mathematical construct for a system in which two salts are being simultaneous drawn down, as it reduces the problem to a single chemical reaction.

Once the starting concentrations for the salts and metals have been estimated, a simple equilibrium equation can be formulated as

$$
K^{\prime}=\frac{x_{C e}^{E Q} y_{L a}^{E Q}}{x_{L a}^{E Q} y_{C e}^{E Q}}
$$

In this equation, $\mathrm{x}$ and $\mathrm{y}$ are mole fractions in salt and metal, respectively. An inherent assumption is made that a metal alloy can form due to the simultaneous reduction of two metals in the salt mixture. If the metal phases existed in pure form, then the metal activity values would be unity and the above equation would be invalid. The equilibrium parameter in this case, $\mathrm{K}^{\prime}$, can be considered to be a lumped parameter defined as follows using fundamental properties.

$$
K^{\prime}=\frac{\gamma_{\mathrm{LaCl} 3} \gamma_{\mathrm{Ce}}}{\gamma_{\mathrm{La}} \gamma_{\mathrm{CeCl} 3}} e^{-\frac{\Delta G^{o}}{R T}}
$$

where $\gamma$ is the activity coefficient, $\Delta \mathrm{G}^{\mathrm{o}}$ is the standard free energy change of reaction, $\mathrm{R}$ is the universal gas constant, 
and $\mathrm{T}$ is the absolute temperature. Here, $\Delta \mathrm{G}^{\circ}$ can be calculated based upon data compiled by Barin [9]. However, activity coefficients are not available in the literature and must be either estimated or determined empirically for each system of interest (requiring representative concentrations). It is, thus, simpler to group activity coefficients with the standard equilibrium constant into the grouped parameter, $\mathrm{K}^{\prime}$. This single parameter can then be determined via fitting experimental data. For the sake of having a consistent convention, the reactions should always be written such that the reactants are more stable thermodynamically than the products.

Note that this model implies that the equilibrium state is independent of the reducing metal. In other words, changing the reducing agent from lithium to calcium or magnesium or sodium or any other active metal should have no effect on the equilibrium state. Provided that the reduction goes to completion, the actual reducing agent is irrelevant to the final outcome. This is an important implication in that it suggests that study of $\mathrm{Ca}, \mathrm{K}, \mathrm{Mg}$ and other potential reducing metals is unnecessary with respect to understanding selectivity.

\section{RESULTS AND DISCUSSION}

It was determined that the lithium reduction occurred very rapidly - within a few hours of initial contact between the salt and lithium. Equilibrium states were estimated based on stabilization of the concentrations of the two reactive chlorides. This equilibrium was usually reached after less than 10 hours of contact between the salt and the lithium. Note that the salt is always being stirred (120 $\mathrm{rpm}$ ) during the experiment to facilitate rapid mass transfer of the lithium throughout the bulk of the salt. It is, thus, possible that samples could be contaminated with fine metal particulate. But the samples appeared to be clean of such contamination. And during dissolution of the salt, metal particulate was not observed. When tests were extended overnight, some unusual long-term behavior was observed. The concentrations of both the primary and secondary chlorides tended to drift significantly away from what had been identified as the equilibrium state. It was postulated that this might have been due to oxygen or water impurities in the atmosphere that could result in transformation of chlorides or metals in the crucible to oxides, oxychlorides, or hydroxides. This could result in either precipitation of metals from the salt phase or redissolution of metals back into the salt phase, effectively masking the ability to observe the drawdown reaction via concentration measurements. It was noted that this drifting of the concentrations occurred when using a glove box with an argon atmosphere containing 20-40 ppm $\mathrm{O}_{2}$ (water was not monitored). Later experiments were carried out in an improved glove box system in which the water and oxygen concentrations were maintained at concentrations of less than $10 \mathrm{ppm}$ each. The

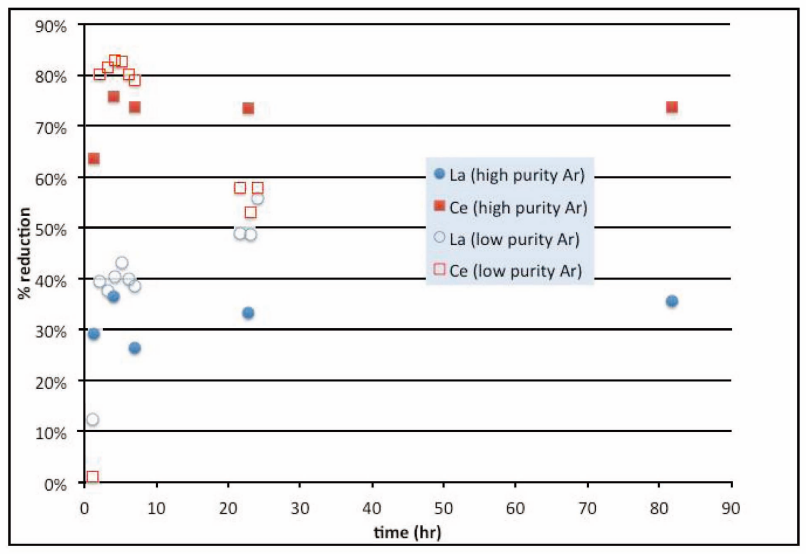

Fig. 2. Transient Drawdown of $\mathrm{CeCl}_{3}-\mathrm{LaCl}_{3}$ in $\mathrm{LiCl}-\mathrm{KCl}$ via Lithium Reduction in High and Low Purity Ar Atmosphere Glovebox Environments.

effect of the change in atmosphere on the salt composition was clearly evident. Salt concentrations were stable for time periods up to $80 \mathrm{hrs}$ using the improved atmosphere. This can be seen in Fig. 2 in which the time-dependent results of $\mathrm{CeCl}_{3}-\mathrm{LaCl}_{3}$ drawdown are shown. For the test in which an uncontrolled atmosphere was used, apparent $\mathrm{CeCl}_{3}$ reduction numbers drop off after about $10 \mathrm{hrs}$ and apparent $\mathrm{LaCl}_{3}$ reduction numbers steadily increase in the same time frame. It is, thus, deduced that any observed long-term instability observed for the active metal chloride concentrations in the salt is due to side reactions with atmosphere impurities. This same concentration instability was observed for several tests that were run for longer than 10 hours using a relatively impure Ar atmosphere.

The primary area of interest for this study was the equilibrium selectivity between the two species being simultaneously reduced. Such data were collected for three salt pairs $\left(\mathrm{LaCl}_{3}-\mathrm{CsCl}, \mathrm{CeCl}_{3}-\mathrm{LaCl}_{3}\right.$, and $\mathrm{NdCl}_{3}$ $\mathrm{LaCl}_{3}$ ). The estimated equilibrium conversion numbers based on experimental measurements are given in Table 1. For each listed test result, the primary species was defined as the one with the least negative free energy of formation, with the difference in standard free energy of formation values for each pair of chlorides is given in Table 1 . The primary species would be the salt compound most likely to be reduced by lithium, thus it may be considered as the target for reduction. In the Cs-La tests, the objective was to reduce varying amounts of $\mathrm{LaCl}_{3}$ while leaving the $\mathrm{CsCl}$ in the salt. Thus, $\mathrm{LaCl}_{3}$ was the primary species. The amount of lithium relative to the reactants (Li Ratio) was varied in these experiments to affect the total extent of reduction. The Li Ratio is specifically defined as the moles of lithium added to the salt per mole equivalent of the primary species. $\mathrm{K}^{\prime}$ is the value for the lumped parameter from the aforementioned model based on a least squares fit of the data. And $\Delta \mathrm{G}$ is the difference in standard free energies of formation of the chloride salt pairs at $500^{\circ} \mathrm{C}$ [9]. 
Table 1. Equilibrium Data from Competitive Lithium Drawdown of Metal Chloride Pairs (Primary and Secondary Species) in LiCl$\mathrm{KCl}$ at $500^{\circ} \mathrm{C}$. $\mathrm{K}^{\prime}$ Values Determined from Model Fits shown in Figure 3. Gibbs Free Energy Differential Based on Thermodynamic Properties Published by Barin [9].

\begin{tabular}{|c|c|c|c|c|c|c|c|}
\hline $\begin{array}{l}\text { Primary } \\
\text { Species }\end{array}$ & $\begin{array}{l}\text { Secondary } \\
\text { Species }\end{array}$ & $\begin{array}{c}\text { Initial } \\
\text { concentration } \\
(\text { mole\% })\end{array}$ & Li ratio & $\begin{array}{c}\text { Primary } \\
\text { Reduction }(\%)\end{array}$ & $\begin{array}{c}\text { Secondary } \\
\text { Reduction }(\%)\end{array}$ & $\mathbf{K}^{\prime}$ & $\begin{array}{c}\Delta G \\
(\mathbf{k J} / \mathbf{m o l} \mathrm{Cl})\end{array}$ \\
\hline \multirow{4}{*}{$\mathrm{LaCl}_{3}$} & \multirow{4}{*}{$\mathrm{CsCl}$} & $2.3 / 2.6$ & 0.28 & 32.1 & 0.81 & \multirow{4}{*}{0.9} & \multirow{4}{*}{73.7} \\
\hline & & $0.41 / 0.80$ & 0.52 & 58.9 & 4.2 & & \\
\hline & & $2.4 / 2.7$ & 0.55 & 60.7 & 4.0 & & \\
\hline & & $0.53 / 0.77$ & 1.0 & 95.6 & 6.6 & & \\
\hline \multirow{5}{*}{$\mathrm{CeCl}_{3}$} & \multirow{5}{*}{$\mathrm{LaCl}_{3}$} & $0.50 / 0.52$ & 1.0 & 81.2 & 39.9 & \multirow{5}{*}{0.2} & \multirow{5}{*}{5.8} \\
\hline & & $2.0 / 2.2$ & 1.0 & 73.6 & 31.7 & & \\
\hline & & $2.2 / 2.3$ & 1.3 & 76.0 & 38.0 & & \\
\hline & & $0.45 / 0.53$ & 1.6 & 93.7 & 79.6 & & \\
\hline & & $0.50 / 0.54$ & 0.28 & 35.9 & 12.5 & & \\
\hline \multirow{4}{*}{$\mathrm{NdCl}_{3}$} & \multirow{4}{*}{$\mathrm{LaCl}_{3}$} & $2.1 / 2.3$ & 0.45 & 41.0 & 14.8 & \multirow{4}{*}{0.26} & \multirow{4}{*}{9.4} \\
\hline & & $0.46 / 0.52$ & 0.77 & 51.9 & 22.9 & & \\
\hline & & $0.44 / 0.53$ & 1.0 & 52.5 & 27.0 & & \\
\hline & & $2.2 / 2.4$ & 1.8 & 97.2 & 98.0 & & \\
\hline
\end{tabular}

The small amount of $\mathrm{CsCl}$ reduction by lithium that was observed was unexpected. Thermodynamically, reaction of $\mathrm{Li}$ with $\mathrm{CsCl}$ is not favorable $\left(\Delta \mathrm{G}_{\mathrm{rxn}}^{\circ}=21.8\right.$ $\mathrm{kJ} / \mathrm{mole}$ ). And in the presence of $\mathrm{La}$ and $\mathrm{LaCl}_{3}$, reduction of $\mathrm{CsCl}$ to $\mathrm{Cs}$ is very unfavorable. It is possible that the reported percentages of reduction were due to experimental error in the measurement of $\mathrm{Cs}$ in the salt samples. In one test, the initial mole fraction of Cs in the salt was measured to be 0.0080 . The subsequent samples taken after $\mathrm{Li}$ was added to the salt had Cs mole fractions ranging from 0.0072 to 0.0080 - a maximum difference of $9 \%$. In the second test involving Cs, no more than $8 \%$ reduction in Cs concentration in the salt was observed. Another possibility is that the decrease in $\mathrm{CsCl}$ concentration is primarily due to an increase in the moles of $\mathrm{LiCl}$ in the salt due to reduction of $\mathrm{LaCl}_{3}$. Calculations indicated that $\mathrm{CsCl}$ concentration can go down by as much as $4.5 \%$ based solely on increase of moles of $\mathrm{LiCl}$ from reduction of $\mathrm{LaCl}_{3}$. The conclusion regarding $\mathrm{CsCl}$ is that it reduces at a very low level if at all in the presence of $\mathrm{Li}$ and $\mathrm{LaCl}_{3}$.

There was found to be no correlation between empirically estimated $\mathrm{K}^{\prime}$ values and the free energy of formation differences. The activity coefficients appear to be highly non-ideal, as the $\mathrm{K}^{\prime}$ values do not compare quantitatively with the standard Gibbs free energy changes of reaction. With the limited amount of data available, it does not appear to be possible to precisely predict $\mathrm{K}^{\prime}$ values. More empirical measurements are likely needed. Future work on the modeling will examine the feasibility to scaling the same mathematical approach to multicomponent (greater than two) systems.

Recall that the ultimate objective is to separate actinides from rare earths, not rare earths from other rare earths. Due to the complexity and cost involved with running experiments with actinides, initial work has focused on rare earthrare earth chloride mixtures as surrogate systems. What has been determined is that the selectivity is qualitatively (if not precisely quantitatively) related to the known fundamental thermodynamic properties. When lithium drawdown is applied to selectively remove actinides from the salt, the free energy gap between the most active actinide $(\mathrm{Am})$ and the least active rare earth $(\mathrm{Y})$ will be only about $3 \mathrm{~kJ} / \mathrm{mole} \mathrm{Cl}$. This corresponds closely to the energy gap between $\mathrm{Ce}$ and $\mathrm{La}$. So, as a first approximation, the $\mathrm{K}^{\prime}$ value between actinides and rare earths can be assumed to be 0.2 . As shown in Fig. 3, it is very difficult to remove a significant amount of the target chloride without also depleting the system of the secondary chloride when $\mathrm{K}^{\prime}$ is only 0.2 . There is some selectivity, but it may be too low to make this a practical process. Further investigation will be pursued to first verify that the selectivity with real actinides is as low as expected and then to assess the impact of low selectivity on the overall process via process simulation. 


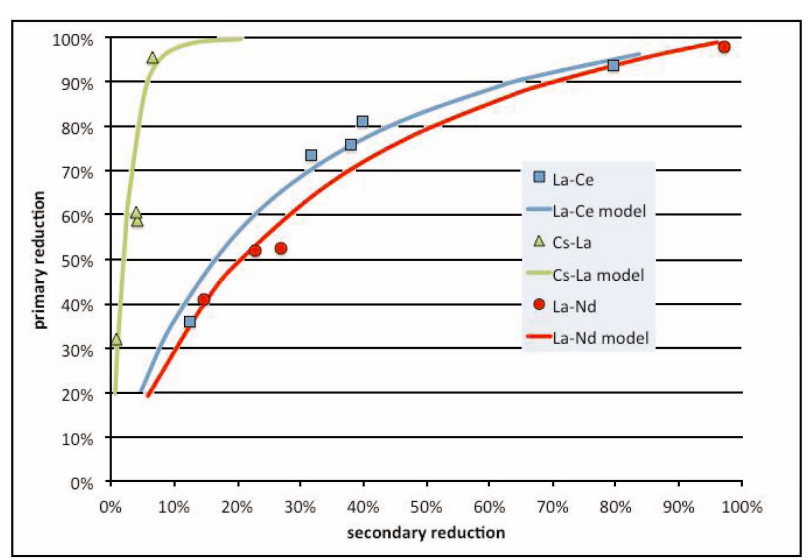

Fig. 3. Fit of Model to Measured Equilibrium Data for Competitive Lithium Drawdown of Metal Chlorides (Primary and Secondary Species) in $\mathrm{LiCl}-\mathrm{KCl}$ at $500^{\circ} \mathrm{C} . \mathrm{K}^{\prime}$ Values were Determined from Visual Estimation and are Included in Table 1.

\section{CONCLUSIONS}

Experiments in which metallic lithium was added to molten chloride salts have examined the viability of using this drawdown method to separate metal chlorides from $\mathrm{LiCl}-\mathrm{KCl}$. Of particular significance, this technique may be applied to separating different metals from waste salt generated from electrorefining spent nuclear fuel based on thermodynamic stability of the chloride forms of the metals. Rapid reduction of the metal chlorides was observed with an apparent equilibrium state being reached in less than $10 \mathrm{hrs}$ after addition of the lithium to the salt. If there was a relatively high level of oxygen and/or water contamination in the glove box's argon atmosphere, concentrations of the metal chlorides drifted away from the equilibrium point in time frames exceeding $10 \mathrm{hrs}$. This is presumably due to reaction to form oxides, oxychlorides, or hydroxides. When the argon atmosphere had $<10 \mathrm{ppm}$ water and oxygen impurities, the metal chloride concentrations were found to be highly stable. Equilibrium selectivity of lithium drawdown was successfully modeled and related to differences in thermodynamic stability of the metal chlorides being reduced to metals. There was found to be significant overlap of reduction for primary and secondary metal chlorides, even with large differences in thermodynamic stability. It is anticipated from these results that there will be significant co-deposition of rare earth metals along with actinides in a real world application of this process. Such co-deposition interferes with the objective of diverting rare earth elements and other waste fission products into the ceramic waste stream without carrying along actinides. Additional testing is still needed using actinide-bearing salt to determine the extent to which the non-ideal selectivity might be a problem.
Inclusion of the equilibrium drawdown model into an overall process model will also be pursued to assess the impact of the lack of high selectivity for this process.

\section{ACKNOWLEDGEMENTS}

The authors wish to acknowledge the invaluable laboratory contributions of Ben Cowan of Idaho National Laboratory and Joanna Taylor of University of Idaho. Support given to this project by the Center for Advanced Energy Studies is also gratefully acknowledged. Funding was provided for this project by Department of Energy's Nuclear Energy Office under the Fuel Cycle Research and Development Program.

\section{REFERENCES}

[ 1 ] K. M. Goff, M. F. Simpson, S. G. Johnson, K. J. Bateman, T. J. Battisti, and S. M. Frank, "Ceramic Waste Form Production and Testing at ANL-West," Proceedings of Third Topical Meeting on DOE Spent Nuclear Fuel and Fissile Material Management, Charleston, SC September 8-11, 1998, American Nuclear Society, 707 (1998).

[2 ] M.F. Simpson, K.M. Goff, S.G. Johnson, K.J. Bateman, T.J. Battisti, K.L. Toews, S.M. Frank, T.L. Moschetti, and T.P. O'Holleran, "A Description of the Ceramic Waste Form Production Process from the Demonstration Phase of the Electrometallurgical Treatment of EBR-II Spent Fuel," Nuclear Technology, 134, 3 (2001).

[ 3 ] K. Kinoshita, T. Inoue, S.P. Fusselman, D.L. Grimmett, J.J. Roy, R.L. Gay, C.L. Krueger, C.R. Nabelek, and T.S. Storvick, "Separation of Uranium and Transuranic Elements from Rare Earth Elements by Means of Multistage Extraction in LiCl-KCl/Be System," Journal of Nuclear Science and Technology, 36, 2 (1999).

[4] M. Kurata, Y. Sakamura, T. Hijikata, and K. Kinoshita, "Distribution behavior of uranium, neptunium, rare-earth elements (Y, La, Ce, Nd, Sm, Eu, Gd) and alkaline-earth metals ( $\mathrm{Sr}, \mathrm{Ba}$ ) between molten $\mathrm{LiCl}-\mathrm{KCl}$ eutectic salt and liquid cadmium or bismuth," Journal of Nuclear Materials, 227, 110 (1995).

[5] J.P. Ackerman and J.L. Settle, "Distribution of Plutonium, Americium, and Several Rare Earth Fission Product Elements Between Liquid Cadmium and LiCl-KCl Eutectic," Journal of Alloys and Compounds, 199, 1-2 (1993).

[6] T. Kobayashi, "An Assessment of the Multi-Stage Counter Current Extraction of TRUs from Spent Molten Salt into Liquid Metal," Journal of Nuclear Science and Technology, 43, 7 (2006).

[ 7 ] J-H. Yoo, H-S. Lee, and E-H. Kim, "Prediction of a Mutual Separation of Actinide and Rare Earth Groups in a Multistage Reduction Extraction System," Nuclear Engineering and Technology, 39, 5 (2007).

[ 8 ] L. S. Chow, J. K. Basco, J. P. Ackerman, and T. R. Johnson, "Molten Salt/Metal Extractions for Recovery of Transuranic Elements," ANL-CP-75688, ANL Symposium on Energy, Environment, and Information Management (1992).

[9] I. Barin, Thermochemical Data of Pure Substances, VCH, Weinheim, New York, 1992. 